\title{
EVALUATION OF PRF MEMBRANE IN ALVEOLAR RIDGE SPLITTING WITH SIMULTANEOUS IMPLANT PLACEMENT (RANDOMIZED CASE CONTROL STUDY)
}

\author{
Omar Sitten*, Mohamed El Mofty ${ }^{* *}$ and Ahmed Gamal ${ }^{* * *}$
}

\begin{abstract}
Aim: To evaluate the use of PRF membrane in alveolar ridge splitting with simultaneous implant placement.

Methodology: 16 patients were randomly selected and treated by alveolar ridge splitting technique to increase bucco-lingual bone width allowing simultaneous implant placement. Divided into 2 groups, case group managed by filling intercortical gap by PRF membrane and control group managed by filling intercortical gap by xenogenic bone graft. Using CBCT to measure buccolingual bone width preoperatively, immediately postoperative and 6 months postoperatively.

Results: Both groups showed a statistically significant increase of the bucco-lingual bone width mean value detected immediately and 6 months postoperatively compared to that measured preoperatively. On the other hand comparing that width detected immediately to that after 6 months postoperatively showed a significant decrease of that value. When comparing bucco-lingual bone width mean values between both groups preoperatively, immediately and 6 months postoperatively were non-significantly different.
\end{abstract}

Conclusions: Using either PRF membrane or xenogenic bone particulates as a filling material in the intercortical gap in alveolar ridge splitting cases could increase buccolingual bone width of the alveolar ridge without significant difference.

\section{KEYWORDS}

Alveolar Ridge Splitting; PRF; Alveolar Ridge Expansion

* Assistant Lecturer of Periodontology, Faculty of Dentistry, MTI University, PhD Degree Candidate, Faculty of Dentistry, Ain Shams University.

** Associate Professor of Oral Medicine, Periodontology, Oral Diagnosis and Radiology Faculty of Dentistry, Ain Shams University, Vice Dean for Community Service and Environmental Development, Faculty of Dentistry, Nahda University.

*** Professor of Oral Medicine, Periodontology, Oral Diagnosis and Radiology, Faculty of Dentistry, Ain Shams University, Vice Dean for Postgraduate Studies and research, 6 October University. 


\section{INTRODUCTION}

Dental implant has been considered as an important solution for replacing missing teeth, however it is guided by the amount of bone available. A thin and atrophic alveolar ridge is considered a challenge to allow successful implant treatment. It is well known that there is a sequnce of bone resorption following tooth extraction over a period of 4 to 12 months following tooth extraction. The buccolingual 1 bone dimension decreases by around half of the original bone width. The bone resorption pattern dimension are more pronounced in the molar regions than in the premolar areas and are even more distinct in the mandible than in the maxilla. Inadequate bone width in the ridge is a common limitation for oral rehabilitation in dental implant therapy. (Araujo et al., 2005)

It is evidenced that we need at least 6 to $7 \mathrm{~mm}$ of horizontal bone width to place an implant with a width of 3.5 to $4 \mathrm{~mm}$ using the conventional drilling protocol. 1 to $1.5 \mathrm{~mm}$ is required on both the buccal and lingual sides of an implant for a successful implant placement. Deficient ridge width less than $5 \mathrm{~mm}$ bucco-lingually require a two-stage surgical protocol. This could be achieved by applying guided bone regeneration procedures to increase ridge width. (Schropp et al., 2003)

Horizontal bone expansion with simultaneous implant placement is used in cases of a thin alveolar ridge to obtain adequate ridge width. This technique has been modified and new methods have been used which include filling of the expanded ridge by different grafting materials. Summers by the use of hand osteotomes invented the ridge expansion technique for implants placed simultaneously. He used these instruments to expand and gradual increase ridge width at the implant site. (Summers et al., 1994)

Micro saw devices and piezoelectric devices are used for cutting and expanding bone of the alveolar ridge under adequate control. Regardless of bone type both devices can be used but with more advantages toward ridges of cancellous bone in the middle. These devices allow preparation of thinner cuts than the conventional burs. Piezoelectric devices allow highly accurate and safe bone cutting using a modulated ultrasonic frequency and copious saline irrigation. Micro vibrations created by piezoelectric device are adjusted only to cut in hard tissue, so allow safety and protection of nerves, vessels and soft tissue. (Suh et al., 2005, Holtzclaw et al., 2010)

Various studies reported the use of graft material between the separated plates and other studies reported not to use any materials. A study carried out by Blus et al., who introduced a technique of expanding the edentulous ridge with no bone grafting material placed in the intercortical gap, which was treated as an extraction socket that require no disturbance. Placement of bone substitutes in intercortical gap has been reported. Advantages of using bone grafts in this gap include internal perfusion, prevention from particle migration and displacement, no need for the donor site and fixation screws and decrease probability of graft resorption. The bone apical to ridge split helps to gain primary stability of the inserted implants. (Blus et al., 2006,

\section{Scipioni et al., 2009)}

Many platelet-derived products or platelet concentrates have been introduced that used as biological mediators to help in the healing mechanism. Platelet-rich fibrin (PRF) is the second product that has proved its efficacy and successful clinical application. It was invented by Choukroun and his team in France. This protocol does not require anticoagulants, bovine thrombin or any other chemicals like PRP. The PRF clot is created by a natural polymerization process during centrifugation that result in natural fibrin network which allow a slow release of growth factors and matrix glycoproteins for 7-14 days during healing process. (Choukroun et al. 2001) 
$\mathrm{PRF}$ is as an autogenous biomaterial which consist of leukocyte and platelet-rich fibrin. It is consisted of three layers: Upper clear colored acellular plasma, red-colored lower segment containing red blood cells (RBCs), and the middle fraction which is the fibrin clot. The platelet and leukocyte distribution is high and rich as we move away from the end of the clot and no more platelets or leukocytes are present beyond the first half of the yellow clot. (Naik et al. 2013)

PRF contain fibrin network and platelets that release growth factors and cytokines which stimulate angiogenesis, clot formation, organization, maturation and have properties of inflammation resistance. The fibrin network formed depends on the polymerization mechanism that happened during centrifugation process that affects it's biological properties. (Dohan et al. 2006)

PRF release different growth factors which are transforming growth factor- $\beta$ (TGF- $\beta$ ) which stimulates proliferation of osteoblasts. Also, it has a role in type 1 collagen synthesis and fibronectin. It improves woven bone formation, stimulates chemotaxis of osteoblast cells and stimulates angiogenesis. The second is platelet-derived growth factor (PDGF) stimulates migration and proliferation of mesenchymal cells and an angiogenic role on endothelial cells. Third is vascular endothelial growth factor (VEGF) which stimulates angiogenesis. Fourth and fifth are Fibroblast growth factor (FGF) and Insulin growth factor-1 (IGF-1) which promote osteoblasts proliferation and have chemotactic effects towards osteoblasts. They Increases the secretion of osteocalcin and aid in the wound healing. Sixth one is Epidermal growth factor (EGF) which enhances cell proliferation, extracellular matrix turnover and has chemotactic effect on periodontal fibroblast cells. (Gassling et al., 2010)

Cone beam computed tomography (CBCT) is a predictable and proved three dimensional (3D) dental and maxillofacial imaging technology that has been improved in recent years. It relies on a process of multiplanar reformation that has several advantages such as minimizing the size of the irradiated area, fast time, image accuracy, special modes of maxillofacial imaging and decreasing of image errors. CBCT has many applications in dentistry including orthodontic treatment planning, temporomandibular joint assessment, extension of cysts and tumors, alveolar ridge before and after placement of dental implant, impacted third molar evaluation and dentoalveolar trauma diagnosis.

\section{(González-García R and Monje F. 2013)}

So our study aimed to evaluate the use of PRF membrane in alveolar ridge splitting associated with simultaneous implant placement.

\section{SUBJECTS, MATERIALS AND METHODS}

This randomized controlled clinical study included sixteen patients who were presenting to us for implant therapy to restore. They were taken randomly from the Outpatient Clinic of Oral Medicine, Periodontology, Oral Diagnosis and Radiology Department, Faculty of Dentistry, Ain Shams University, Cairo, Egypt. This study was conducted from the period from September 2016 to August 2019. An Ethical approval of the study was obtained from the Ethical Committee of Faculty of Dentistry, Ain- Shams University, Egypt.

Patients were selected randomly into 2 main groups using computer generated randomization* and was performed by another individual other than the investigator. Each patient was given a certain number. The purpose and technical steps of the study were explained and signed in a written informed Arabic consent that were given to all patients.

The number of patients and sample size calculation were done according to Sample Size

\footnotetext{
* www.randomizer.org
} 
program* for parallel group clinical trials with binary data. Thus, for a study power of $84 \%$ and alpha $(\alpha)$ level of $0.0,16$ cases would be divided into 2 arm groups ( 8 patients for each group). The study consists of two groups:

Group 1 (8 patients): This group had alveolar ridge splitting to increase bone width and simultaneous implants placement ${ }^{* *}$ and using PRF membrane to fill intercortical gap and covered by collagen membrane ${ }^{* * *}$.

Group 2 (8 patients): To these patients we applied the same method of alveolar ridge splitting and simultaneous implants placement and using xenogenic bone graft $^{* * * *}$ to fill intercortical gap covered by collagen membrane.

Patients included in this study were from both genders with age range (18 -50) years, good compliance with the plaque control instructions, patients have maxillary edentulous anterior/ premolar area with an average width of the ridge bone of 3 to $4 \mathrm{~mm}$, and a minimum of $11 \mathrm{~mm}$ of bone height, including at least $1 \mathrm{~mm}$ of cancellous bone to gain bone width and with availability for recall. The excluded patients were uncontrolled diabetic patients with HBA1c > 7, uncontrolled cardiac patient or patients in anticoagulant therapy, osteoporotic patients taking Bisphosphonates therapy, patients suffering from active periodontal disease, smoking habit, presence of any periapical or bone pathology adjacent to implant site and pregnant females.

\section{Clinical evaluation}

Clinical evaluation of each of the following was done preoperatively, at time of implants insertion, 6 months and 9 months post-operative.

\section{Gingival index (GI): (Loe 1967)}

Grade 0: Normal gingiva with no erythema and bleeding.

Grade 1: Mild inflammation, mild surface alteration and no bleeding.

Grade 2: Moderate inflammation, moderate erythema, swelling and bleeding on probing or when pressure applied.

Grade 3: Severe inflammation, severe erythema, swelling and tendency toward spontaneous bleeding.

\section{Plaque index (PI): (Silness and Loe 1964)}

Grade 0: With no plaque is present on the teeth.

Grade 1: No visible plaque but detected by probe.

Grade 2: Visible plaque forms a thin continuous band at the cervical margin of the tooth.

Grade 3: Visible plaque forms a band covering more than the cervical third of the crown of the tooth.

\section{Radiographic Evaluation}

CBCT scans were taken (for the segment which includes the implant site to reduce patient's exposure dose as possible), immediately after surgery and 6 months postoperatively using CBCT machine ${ }^{* * * * *}$ at the Oral Radiology Department, Faculty of Dentistry, Ain-shams University. Parameters was $0.2 \mathrm{~mm}$ voxel size and $6 \times 8 \mathrm{~cm}$ field of view. To optimize the viewing condition, before interpretation and measurements, aligned protocol settings were done "re-orientation of the orthogonal images was done so that the sagittal plane (Y) is oriented parallel to the long axis of the implant under examination.

\footnotetext{
* (Version 1.1.3 Sheffield, UK: Epigenesys) developed by Julious \& Campell 2012

** Neo CMI implant, Neobiotech Company, South Korea.

*** Hypro Sorb Collagen Membrane, Bioimplon GmbH, Friedrich-List-Str. 27, 35398 Giessen, Germany.

***** Bovine bone, BioOss Natural Bone Grafting Material, Geistlich Biomaterials Company.

****** I-CAT machine, Imaging Sciences International, Hatfield, PA, USA.
} 
When this was done, automatically the coronal plane would be set perpendicular to the long axis of the implant to measure bucco-palatal bone width:

The bucco-palatal bone thickness of the alveolar bone was measured after selecting the crosssectional image made at the midpoint of ridge crest preoperatively or postoperatively at the implant.

\section{Patient pre-surgical preparation}

1. Scaling and root planning were performed and oral hygiene instructions were given to the patient including teeth brushing, dental flossing and prophylactic use of antibacterial mouthwash $0.12 \%$ chlorhexidine gluconate.

2. One day before surgery patient was instructed to take prophylactic dose of antibiotic include $1 \mathrm{gm}$ Amoxicillin tablet and $500 \mathrm{mg}$ metronidazole tablet 2 times daily that were continued to full course after surgery for 7 days.

\section{- Surgical procedures:}

1. Anesthesia Lidocaine hydrochloride $2 \%$ epinephrine, $1 / 100000 *$ was administrated at the surgical site.

2. A para crestal incision was made, with a surgical blade $(15 \mathrm{c}) *$ on palatal side of the crest of the edentulous ridge crest with one or two vertical releasing incision.

3. A full-thickness flap was raised to obtain adequate visibility of the crestal bone.

4. The osteotomy was done on midcrestal bone in the form of horizontal cut with a bone microsaw** to separate the buccal plate from the palatal plate, and was limited to $1 \mathrm{~mm}$ from the adjacent teeth.

5. One or two vertical cuts on both side of horizontal cut to allow separation of bone plates without fracture, and were $2 \mathrm{~mm}$ short of the implants length.
6. Bone chisels and mallet were used to separate the buccal plate from the palatal plate by gentle tapping and mesiodistal movement of chisels to a depth corresponding to implants length.
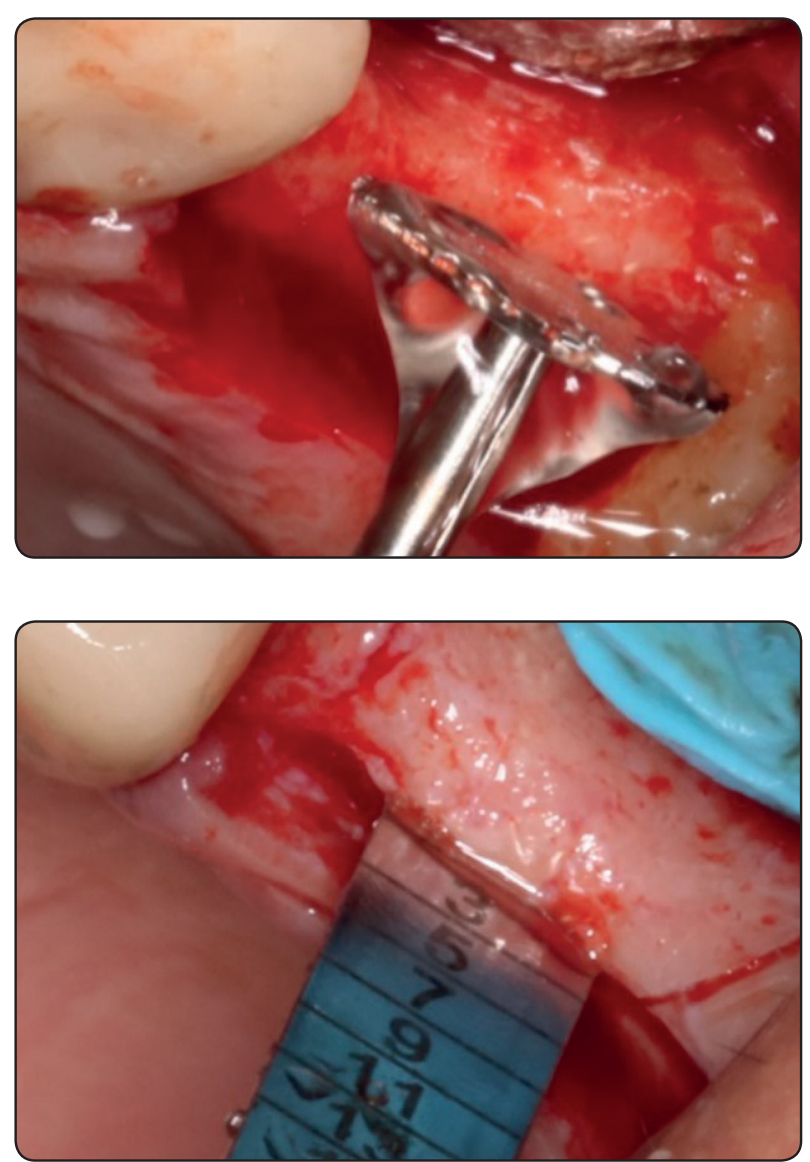

7. Osteotomy sequential drilling was done using starter drill followed by pilot drill in accurate locations for implant placement $1.5 \mathrm{~mm}$ from adjacent natural teeth and $3 \mathrm{~mm}$ from adjacent implants.

8. Using bone expanders to complete osteotomy and create a compatible diameter width compatible with implant width.

9. After ridge expansion, implants were placed $1 \mathrm{~mm}$ subcrestal to compensate for crestal bone resorption associated with ridge splitting procedures, and all cover screw implants were left submerged. 

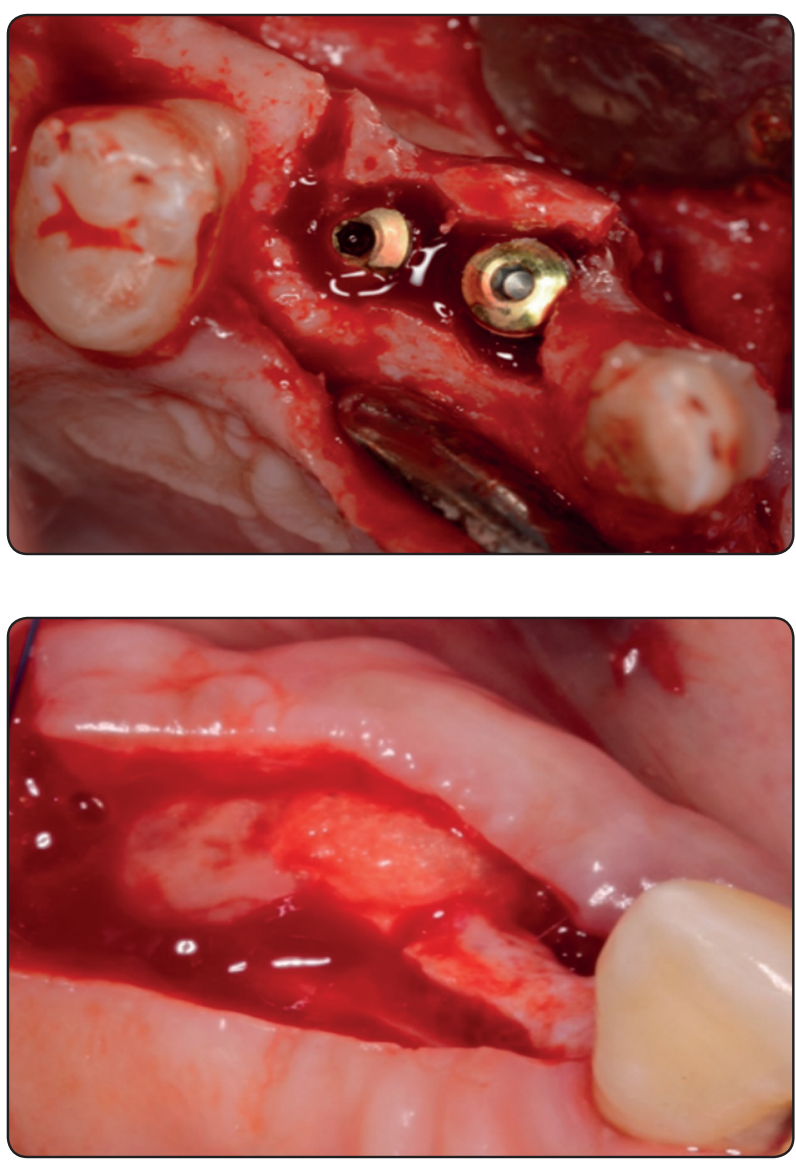

10. In the first group, PRF was obtained by taking a $10 \mathrm{ml}$ blood sample from the vein patient in a glass vacuumed tube without anticoagulant.

11. Movement of the glass tube to the centrifuge for 10 minutes at $3000 \mathrm{rpm}$, then will be left for 10 minutes in the centrifuge for complete coagulation of the fibrin membrane.

12. Using tissue forceps to collect PRF membrane from the intermediate layer in the tube (buffy coat), and putt the PRF clot in a sterile cup for approximately 10 minutes to allow release of the proper serum contained within.

13. PRF Clot was transformed into a membrane through the compression between two sterile gauzes wetted with saline.

14. PRF membrane was placed in the intercortical gap between implants then covered by collagen membrane.
15. In the second group, xenogeneic particulate bone graft mixed with saline was placed in the intercortical gap then covered by collagen membrane.

16. The flap was sutured passively without tension with intimate contact between flap edges to protect the implants and grafting material.

\section{- Postoperative treatment steps:}

1. Antibiotic (Amoxcillin 1 gm every 12 hours, metronidazole $500 \mathrm{mg}$ every 12 hours), antiinflammatory (Alphintern tablets every 8 hours) and analgesics (Ibuprofen $600 \mathrm{mg}$ every 12 hours) were prescribed for the patient for 7 days and instructed to avoid brushing at the surgical site only rinsing with warm saline and $0.12 \%$ chlorohexidine mouthwash.

2. Patient was called after a week for follow up and ensuring good oral hygiene and sutures were removed after 2 weeks.

3. After 6 months of healing, stage-two surgery was carried out by exposures of implants and placing of appropriate healing abutments.

\section{Statistical analysis}

All data was collected blindly and subjected to statistical analysis of data which was done using the software statistical package SPSS program version 17. Chi square test, Fisher exact test and odds ratio (with the $95 \%$ confidence interval) were used to compare the frequency of genotypes in different studied groups. In addition, Hardy-Weinberg test was used to test for genetic equilibrium among studied cases and controls. A p-value of less than 0.05 indicated a significant level. Qualitative variable was described as number.

\section{RESULTS}

In our study we recruited 16 Egyptian patients complaining from edentulous alveolar ridge with horizontal bone defect but had sufficient vertical bone height and soft tissue thickness. They managed 
by alveolar ridge splitting technique to increase bucco-lingual bone width allowing simultaneous implant placement. For ridge splitting we used bone micro-saw for horizontal and vertical bony cuts followed by bone chisels and expanders. Patients have divided into 2 groups with different treatment modality to fill intercortical gap. In group 1 we used PRF membrane to fill intercortical gap and covered by collagen membrane, while in group 2 used xenogenic bone graft to fill intercortical gap then covered by collagen membrane. We monitored the changes and the effect of ridge splitting technique with simultaneous implant placement on the horizontal bone width in our studied groups.

Table 1 shows descriptive data of all cases with horizontal bone defect managed by ridge splitting procedure with simultaneous implant placement (16 cases in 2 groups, 8 in each group). Their age mean was $37.5 \pm 8.7$ years ranging from 23 to 53 years old. They were in the form of 9 females and 7 males. Their bucco-lingual bone width as assessed by CBCT at presentation showed a mean value of $4.23 \pm 0.58 \mathrm{~mm}$ which is preventing straight forward implant placement, so ridge splitting procedure was indicated with simultaneous implant placement.
CBCT immediate postoperative picture showed an increase in bucco-lingual bone width with mean value $7.72 \pm 0.69 \mathrm{~mm}$ followed by a decrease at 6 months postoperatively with a mean value of 7.36 $\pm 0.68 \mathrm{~mm}$.

Plaque index of both groups of implant candidate cases with horizontal bone defect preoperatively as well as 6 months postoperatively. From this table, the plaque index score of most cases was 1 preoperatively (12/16, 6 in each group). Follow up after 6 months postoperatively showed an index of 1 in 12 cases (7/8 in group I and 5/8 in group II). There was a decrease in the plaque index postoperatively from that preoperatively with no significant difference between both groups ( $p>0.05)$. Gingival index of both groups of implant candidate cases with horizontal bone defect preoperative as well as 6 months postoperatively. From this table, the gingival index of most cases was 1 preoperatively $(9 / 16,4$ in group I and 5 in group II). Follow up after 6 months postoperatively showed an index of 1 in 13 cases (7/8 in group I, and 6/8 in group II). There was a decrease in the gingival index postoperatively from that preoperatively with no significant difference between both groups ( $p>0.05)$.

TABLE (1) Descriptive data of all studied cases (16 cases) with horizontal bone defect managed by ridge splitting procedure with simultaneous implant placement:

\begin{tabular}{|c|c|c|c|c|}
\hline & Mean \pm SD & Min & Max & Median \\
\hline Age & $37.5 \pm 8.7$ & 23.0 & 53.0 & 39.5 \\
\hline \multicolumn{5}{|l|}{ Bucco-lingual Bone width } \\
\hline Preoperative & $4.23 \pm 0.58$ & 3.4 & 5.20 & 4.150 \\
\hline Postoperative immediately & $7.72 \pm 0.69$ & 6.40 & 9.20 & 7.700 \\
\hline Postoperative 6 months & $7.36 \pm 0.68$ & 6.00 & 8.80 & 7.350 \\
\hline \multirow[t]{2}{*}{ Gender $M / F(N)$} & Group I & Group II & $\mathbf{X} 2$ & $\mathbf{p}$ \\
\hline & $\mathrm{N}=8$ & $\mathrm{~N}=8$ & & \\
\hline Female & 5 & 4 & 0 & 0.5 \\
\hline Male & 3 & 4 & & \\
\hline
\end{tabular}


Table 2 shows follow up results of bucco-lingual bone width in group I preoperatively as well as immediately and 6 months postoperatively and their statistical significance. This table shows a statistically significant increase of the mean value of bucco-lingual bone width detected preoperatively compared to that measured immediately and 6 months postoperatively $(4.21 \pm 0.58$ vs. $8.04 \pm$ $0.71,4.21 \pm 0.58$ vs. $7.68 \pm 0.69$, respectively, $\mathrm{p}=$ $0.000, p=0.000$ respectively). On the other hand comparing the bucco-lingual bone width that was detected immediately postoperatively to that after 6 months showed a significant decrease of that value $(8.04 \pm 0.71$ vs. $7.68 \pm 0.69, p=0.037)$.

Table 3. Shows follow up results of buccolingual bone width in group II preoperatively as well as immediately and 6 months postoperatively and their statistical significance. This table shows a statistically significant increase of the mean value of bucco-lingual bone width detected immediately and 6 months postoperatively compared to that measured preoperatively $(4.25 \pm 0.61$ vs. $7.41 \pm$ $0.55,4.25 \pm 0.61$ vs. $7.05 \pm 0.56$ respectively, $\mathrm{p}=$ $0.000, p=0.000$ respectively). On the other hand comparing the bucco-lingual bone width that was detected 6 months postoperatively to that measured immediately showed a significant decrease of that value $(7.41 \pm 0.55$ vs. $7.05 \pm 0.56,, p=0.000$.

Table 4. Shows comparison between both groups regarding all studied parameters. From this table, the age mean of group I was $37.38 \pm 9.27$ which was non-significantly different from that of group II $(37.63 \pm 8.75, \mathrm{p}=0.95)$. The primary implant stability mean value and SD was the same in both groups $(27.50 \pm 4.63, p=1)$. Regarding the buccolingual bone width, the mean value preoperatively $(4.21 \pm 0.58$ vs. $4.25 \pm 0.61 \mathrm{p}=0.885)$ immediately $(8.04 \pm 0.70$ vs. $7.41 \pm 0.55 \mathrm{p}=0.066)$ and 6 months postoperatively $(7.68 \pm 0.69$ vs. $7.05 \pm 0.56 \mathrm{p}=$ $0.065)$ were non-significantly different between both groups.

TABLE (2) Follow up results of bucco-lingual bone width in group I preoperatively as well as immediately and 6 months postoperatively and their statistical significance:

\begin{tabular}{cccc}
\hline Bucco-lingual Bone width & Mean \pm SD & Mean \pm SD & p \\
\hline Preoperative vs. Postoperative immediately & $4.21 \pm 0.58$ & $8.04 \pm 0.71$ & $0.000^{* *}$ \\
\hline Preoperative vs. Postoperative 6 months & & $0.000^{* *}$ \\
\hline Postoperative immediately vs. 6 months & $8.08 \pm 0.69$ & $7.68 \pm 0.69$ & $0.037^{*}$ \\
\hline
\end{tabular}

Significant $* p<0.05 * *<<0.001$

TABLE (3) Follow up results of bucco-lingual bone width in group II preoperatively as well as immediately, 6 and 9 months postoperatively and their statistical significance:

\begin{tabular}{clll}
\hline Bucco-lingual Bone width & Mean \pm SD & Mean \pm SD & p \\
\hline Preoperative vs. Postoperative immediately & $4.25 \pm 0.61$ & $7.41 \pm 0.55$ & $0.000^{* *}$ \\
\hline Preoperative vs. Postoperative 6 months & $4.25 \pm 0.61$ & $7.05 \pm 0.56$ & $0.000^{* *}$ \\
\hline Postoperative immediately vs. 6 months & $7.41 \pm 0.55$ & $7.05 \pm 0.56$ & $0.000^{* *}$ \\
\hline
\end{tabular}


TABLE (4) Comparison of mean value of studied parameters in both groups with horizontal bone defect managed by ridge splitting with simultaneous implant placement:

\begin{tabular}{cccc}
\hline Studied Parameter & $\begin{array}{c}\text { Group I } \\
\mathrm{N}=8\end{array}$ & $\begin{array}{c}\text { Group II } \\
\mathrm{N}=8\end{array}$ & \\
\hline & Mean $\pm \mathrm{SD}$ & Mean $\pm \mathrm{SD}$ & P t-test \\
\hline Age & $37.38 \pm 9.27$ & $37.63 \pm 8.75$ & 0.957 \\
\hline 1ry implant stability & $27.50 \pm 4.63$ & $27.50 \pm 4.63$ & 1.000 \\
\hline Preoperative & Bucco-lingual Bone width & & \\
\hline Postoperative immediately & $4.21 \pm 0.58$ & $4.25 \pm 0.61$ & 0.885 \\
\hline Postoperative 6 months & $8.04 \pm 0.70$ & $7.41 \pm 0.55$ & 0.066 \\
\hline & $7.68 \pm 0.69$ & $7.05 \pm 0.56$ & 0.065 \\
\hline
\end{tabular}

Significant $* p<0.05 * * 0<0.001$

TABLE (5) Comparison between both groups regarding value of change of Bucco-lingual bone width difference immediately and 6 months postoperatively

\begin{tabular}{|c|c|c|c|}
\hline & $\begin{array}{c}\text { Group I } \\
N=8 \\
\text { Mean } \pm S D\end{array}$ & $\begin{array}{c}\text { Group II } \\
N=8 \\
\text { Mean } \pm S D\end{array}$ & $P$ \\
\hline \multicolumn{4}{|c|}{ Bucco-lingual bone width difference } \\
\hline Postoperative immediately $\underline{\text { vs. }} 6$ months & $0.36 \pm 0.40$ & $0.36 \pm 0.13$ & 0.967 \\
\hline
\end{tabular}

Significant $* p<0.05 * * * 0.001$

Table 5. Shows comparison between both groups regarding value of change of Bucco-lingual bone width difference immediately and 6 months postoperatively. From this table, the difference of the bucco-lingual bone width in the time between immediate and 6 months postoperatively was $0.36 \pm$ $0.40 \mathrm{~mm}$ in group I and $0.36 \pm 0.13 \mathrm{~mm}$ in group II with no statistical significance $(\mathrm{p}=0.967)$.

\section{DISSCUSION}

For augmentation of horizontal bone width, ridge splitting is considered one of the predictable treatment options. It is applied and indicated for expansion of insufficient alveolar ridge width but has adequate vertical height. Also, it required some intervening cancellous bone present between buccal and lingual cortex and without bony undercuts to decrease risk of ridge fracture. During this technique for optimal implant initial stability, implants are placed apical to vertical bony cuts. The primary advantages of this technique are reduced tissue morbidity due to lack of the second surgical donor site, reduced treatment time and one stage augmentation procedure which are the main drawbacks of autogenous bone block graft.

In this study we recruited 16 Egyptian patients complaining from edentulous alveolar ridge with horizontal bone defect but had sufficient vertical bone height and soft tissue thickness. They managed by alveolar ridge splitting technique to increase 
bucco-lingual bone width allowing simultaneous implant placement. For ridge splitting we used bone micro-saw for horizontal and vertical bony cuts followed by bone chisels and expanders. The using of this technique was in accordance with Dene et al., 2010 demonstrated using of hand osteotomes results in successful expansion of the alveolar ridge at a single implant site. A very pleasing final esthetic result was achieved by this technique and allows for immediate implant placement. Cortese et al., 2016 who postulated that piezosurgery was avoided due to high sensitivity during surgical bone preparation. Also, using osteotomes allow to detect the difference in consistency between the trabecular and cortical bone which is not always associated with piezosurgery and increasing the risk of fenestration of the vestibular cortex.

Patients have divided into 2 groups with different treatment modality to fill intercortical gap. In group 1 we used PRF membrane to fill intercortical gap and covered by collagen membrane, while in group 2 used xenogenic bone graft to fill intercortical gap then covered by collagen membrane. We monitored the changes and the effect of ridge splitting technique with simultaneous implant placement on the soft tissue thickness, horizontal bone width and the marginal bone loss in our studied groups.

Application of PRF membrane in in implant placement reported by Hamzacebi et al., 2015 indicated that treatment using PRF in peri-implant defects cases was clinically more effective than with access flap surgery alone. Regardless of the defect morphology, PRF showed that it is an effective, practical and inexpensive autologous blood-clotting medium. Also, it may also enhance the results of surgical peri-implantitis managment. Also, Liu et al., 2019 Research findings indicated that PRF as a bone filling material is a promising treatment modality for oral and maxillofacial bone regeneration. PRF has been proved to improve differentiation, proliferation, migration and mineralization of cells during bone formation with variations according to the cell type. However, PRF alone has an unstable effect on bone regeneration.

Regarding the bucco-lingual bone width of edentulous alveolar ridge measured by $\mathrm{CBCT}$ in group I, shows a statistically significant increase of the mean value of bucco-lingual bone width detected immediately and 6 months postoperatively compared to that measured preoperatively. On the other hand comparing the bucco-lingual bone width that was detected immediately postoperatively to that after 6 months showed a significant decrease of that value. Also, in group II shows a statistically significant increase of the mean value of buccolingual bone width detected immediately and 6 months postoperatively compared to that measured preoperatively. On the other hand comparing the bucco-lingual bone width that was detected immediately postoperatively to that after 6 months showed a significant decrease of that value. When comparing bucco-lingual bone width mean values between both groups preoperatively, immediately and 6 months postoperatively were non-significantly different. Indicating that ARS was effective treatment modality for increasing ridge width and allow simultaneous implant placement but there was some decrease in that width gained after complete healing and implant loading especially in xenogenic bone graft group.

The buccolingual width gained is in accordance with Bassetti et al., 2015 who reported a systematic review to evaluate clinical, histological and radiological outcomes of the alveolar ridge splitting/ expansion technique with or without GBR. They reported that ARST seems to be a predictable onestage alternative to long time two-stage horizontal augmentation procedures. Waechter et al., 2016 reported in a systematic review that the alveolar ridge splitting technique seems to be a predictable and effective procedure to gain bone width, regardless of the surgical instruments used.

Similar to our study Nguyen et al., 2016 reported immediate gain in bone width may not be permenant and some decrease in this gain may occur over time 
and this is affected by different factors including bone compression during expansion, surgical instrumentation, flap reflection and implant system used. Also, Jensen and Becktor 2019 systematic review reported that split-crest technique appears to be predictable for horizontal augmentation of maxillary alveolar width defects with high implant survival percentage. de Souza et al., 2020 concluded that the split crest technique proved to be effective and predictable treatment modality results in a large increase in ridge width and a high rate of implant survival.

In the end in our study we concluded that ARS technique with simultaneous implant placement is effective and successful treatment modality for ridge expansion and horizontal augmentation. Using either PRF membrane or xenogenic bone particulates as a filling material in the intercortical gap in alveolar ridge splitting cases could increase buccolingual bone width of the alveolar ridge without significant difference.

\section{REFERENCES}

1. Araujo MG, Lindhe J. Ridge alterations following tooth extraction with and without flap elevation: An experimental study in the dog. Clin Oral Implants Res 2009;20:545-549.

2. Araujo MG, Lindhe J. Ridge alterations following tooth extraction with and without flap elevation: An experimental study in the dog. Clin Oral Implants Res 2009;20:545-549.

3. Bassetti MA, Bassetti RG, Bosshardt DD. The alveolar ridge splitting/expansion technique: a systematic review. Clin Oral Implants Res. 2016 Mar;27(3):310-24. doi: 10.1111/clr.12537. Epub 2015 Jan 14. PMID: 25586966.

4. Choukroun J, Diss A, Simonpieri A, Girard MO, Schoeffler C, Dohan SL, et al. Platelet-rich fibrin (PRF):A second-generation platelet concentrate, Part IV: Clinical effects on tissue healing. Oral Surg Oral Med Oral Pathol Oral Radiol Endod. 2006; 101:E56-60.

5. Cortese A, Pantaleo G, Amato M, Claudio PP. Ridge Expansion by Flapless Split Crest and Immediate Implant Placement: Evolution of the Technique. J Craniofac Surg. 2016Mar;27(2):e1238.doi:10.1097/SCS.0000000000002367.
6. de Souza CSV, de Sá BCM, Goulart D, Guillen GA, Macêdo FGC, Nóia CF. Split Crest Technique with Immediate Implant to Treat Horizontal Defects of the Alveolar Ridge: Analysis of Increased Thickness and Implant Survival. J Maxillofac Oral Surg. 2020 Dec;19(4):498-505. doi: 10.1007/s12663-020-01332-z. Epub 2020 Jan 18. PMID: 33071495

7. Dene L, Condos S. Ridge expansion and immediate implant placement in the esthetic zone. N Y State Dent J 2010;76(2):28-31.

8. Dohan DM, Choukroun J, Diss A, , Dohan SL, Dohan

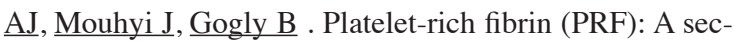
ond-generation platelet concentrate. Part I: Technological concepts and evolution. Oral Surg Oral Med Oral Pathol Oral Radiol Endod 2006; 101:e37-e44.

9. González-García R, Monje F. The reliability of cone-beam computed tomography to assess bone density at dental implant recipient sites: a histomorphometric analysis by micro-CT. Clin Oral Implants Res. 2013 Aug;24(8):871-9.

10. Hamzacebi B, Oduncuoglu B, Alaaddinoglu EE. Treatment of Peri-implant Bone Defects with Platelet-Rich Fibrin. Int J Periodontics Restorative Dent. 2015 MayJun;35(3):415-22. doi: 10.11607/prd.1861. PMID: 25909530

11. Holtzclaw DJ, Toscano NJ, Rosen PS. Reconstruction of posterior mandibular alveolar ridge deficiencies with the piezoelectric hingeassisted ridge split technique: A retrospective observational report. J Periodontol 2010;81:15801586.

12. Julious SA, Campell MJ. Tutorial in biostatistics: sample sizes for parallel group clinical trials with binary data. Stat Med. 2012 Oct 30; 31(24):2904-36.

13. Liu Y, Sun X, Yu J, Wang J, Zhai P, Chen S, Liu M, Zhou Y. Platelet-Rich Fibrin as a Bone Graft Material in Oral and Maxillofacial Bone Regeneration: Classification and Summary for Better Application. Biomed Res Int. 2019 Dec 6;2019:3295756. doi: 10.1155/2019/3295756. PMID: 31886202; PMCID: PMC6925910.

14. Löe H. The Gingival Index, the Plaque Index and the Retention Index Systems. J Periodontol. 1967 Nov-Dec; 38(6): Suppl:610-6.

15. Naik B, Karunakar P, Jayadev M, Marshal VR. Role of Platelet rich fibrin in wound healing: A critical review. J Conserv Dent.2013 Jul; 16(4):284-93. 
16. Nguyen VG, von Krockow N, Weigl P, Depprich R. Lateral Alveolar Ridge Expansion in the Anterior Maxilla Using Piezoelectric Surgery for Immediate Implant Placement. Int J Oral Maxillofac Implants. 2016 May-Jun;31(3):687-99. doi: 10.11607/jomi.4214. PMID: 27183077

17. Schropp L, Wenzel A, Kostopoulos L, Karring T. Bone healing and soft tissue contour changes following singletooth extraction: A clinical and radiographic 12-month prospective study. Int $\mathrm{J}$ PeriodonticsnRestorative Dent 2003;23:313-323.3.

18. Scipioni A, Calesini G, Micarelli C, Coppè S, Scipioni L. Morphogenic bone splitting: Description of an original technique and its application in esthetically significant areas. Int J Prosthodont 2008;21:389-97.

19. Silness J \& Loe H. Periodontal disease in pregnancy II Correlation between aral hygiene and periodontal condition. Acta Odontal Scand 1964; 22: 121-35.

20. Starch-Jensen T, Becktor JP. Maxillary Alveolar Ridge Expansion with Split-Crest Technique Compared with
Lateral Ridge Augmentation with Autogenous Bone Block Graft: a Systematic Review. J Oral Maxillofac Res. 2019 Dec 30;10(4):e2. doi: 10.5037/jomr.2019.10402. PMID: 32158526; PMCID: PMC7012616.

21. Suh JJ, Shelemay A, Choi SH, Chai JK. Alveolar ridge splitting: A new microsaw technique. Int J Periodontics Restorative Dent 2005;25: 165-171.

22. Summers RB. The osteotome technique: Part 2-The ridge expansion osteotomy (REO) procedure. Compendium 1994;15:422, 424, 426.

23. V. Gassling, T. Douglas, P. H. Warnke, Y. Ac,il, J. Wiltfang, and S. T. Becker; Platelet-rich fibrin membranes as scaffolds for periosteal tissue engineering,Clinical. Oral Implants Research; 2010; 21; 543-549.

24. Waechter J, Leite FR, Nascimento GG, Carmo Filho LC, Faot F. The split crest technique and dental implants: a systematic review and meta-analysis. Int $\mathrm{J}$ Oral Maxillofac Surg. 2017 Jan;46(1):116-128. doi: 10.1016/j. ijom.2016.08.017. Epub 2016 Sep 14. PMID: 27639295 